\title{
Uterine artery embolization in residual arterio-venous fistula due to invasive mole using glue and polyvinyl alcohol particles: Case report
}

\author{
Kishor Taori, Jawahar Rathod, Amit Disawal, Anand Hatgaonkar, Suresh Dhakate, Ramesh Parate, \\ Shyam Chhadi, Vishal Bakare, Prasad Wavare, Rakhi Puria
}

Department of Radio-Diagnosis, Government Medical College and Hospital, Nagpur, India

Email: kishortaori@gmail.com

Received 5 December 2012; revised 7 January 2013; accepted 15 January 2013

\begin{abstract}
Gestational trophoblastic diseases (GTD) are one of the rare complications of pregnancy, which include partial and complete moles with their more aggressive counterparts termed as Gestational trophoblastic neoplasias (GTN). Their association with uterine vascular malformations is well recognised and should be suspected in appropriate clinical settings. Selective uterine artery embolization is a safe and effective alternative method of treatment in a patient with severe per vaginal bleeding from residual uterine vascular malformations due to GTN which has relative lower morbidity and mortality than surgical procedures. Here, we report a case of residual uterine arterio-venous malformation due to invasive mole detected by ultrasonography (US) and contrast enhanced MRI \& referred to Interventional Radiology for Transcathetor arterial embolization.
\end{abstract}

Keywords: Gestational Trophoblastic Diseases and Neoplasias; Glue; Polyvinyl Alcohol Particles; Selective Uterine Artery Embolization; Vascular Malformation

\section{INTRODUCTION}

Gestational trophoblastic diseases include complete and partial mole formation, however have rare occurrence rate $[1,2]$. Approximately $10 \%$ of complete hydatidiform moles and $0.5 \%$ of partial hydatidiform moles undergo malignant transformation into an invasive mole, a choriocarcinoma, or, rarely, a placental site tumor, and require further treatment, and are collectively known as Gestational Trophoblastic Neoplasias with majority of them treated by chemotherapy [2,3]. GTN are highly vascular and are associated with the formation of uterine vascular malformations. The rate of persistence of these vascular malformations is about $10 \%-15 \%$, even after complete resolution of the tumour following chemotherapy [4].
Overall, $1 \%-2 \%$ of these uterine vascular malformations cause vaginal or rarely intraperitoneal haemorrhage, which can be life threatening. The traditional treatment of choice was hysterectomy and uterine artery ligation for achieving haemostasis. However, the advent of selective uterine artery embolization has provided a minimally invasive and more effective alternative strategy for the management of these patients. Here, we report a case of residual uterine vascular malformation due to invasive mole which was successfully treated with selective bilateral uterine artery embolization using glue and polyvinyl alcohol particles.

\section{CASE REPORT}

A 32 yrs old female with complaints of bleeding per vagina on and off since 1 month, was referred for US pelvis examination. She had history of 3 months amenorrhea. On clinical examination of the abdomen, there was mild tenderness. Her blood picture revealed features of anaemia ( $\mathrm{Hb}: 5.5 \mathrm{gm} \%$ ), and her urine pregnancy test was strongly positive. US of the pelvis revealed bulky uterus with heterogeneous appearing internal contents and loss of endo-myometrial interface at places (Figure 1). On Doppler study, there was significant neovascularity seen within the lesion with reduction in the resistivity index (Figure 2). Laboratory tests revealed raised serum B hCG levels $(13565.3 \mathrm{mIU} / \mathrm{mL})$. On correlating the clinical, biochemical and US findings, the diagnosis of Molar pregnancy was given. Chest X ray was then done which showed bilateral clear lung fields. Patient was advised MRI pelvis for further evaluation of the lesion. It revealed bulky uterus with presence of solid cystic lesion within the endometrial cavity showing multiple flow voids (Figures 3 and 4) and heterogeneous contrast enhancement (Figures 5 and 6). The lesion showed invasion into the myometrial tissue at multiple sites (Figure 7) and was seen to closely abut the posterior wall of urinary bladder. There was no obvious parametrial spread. On 


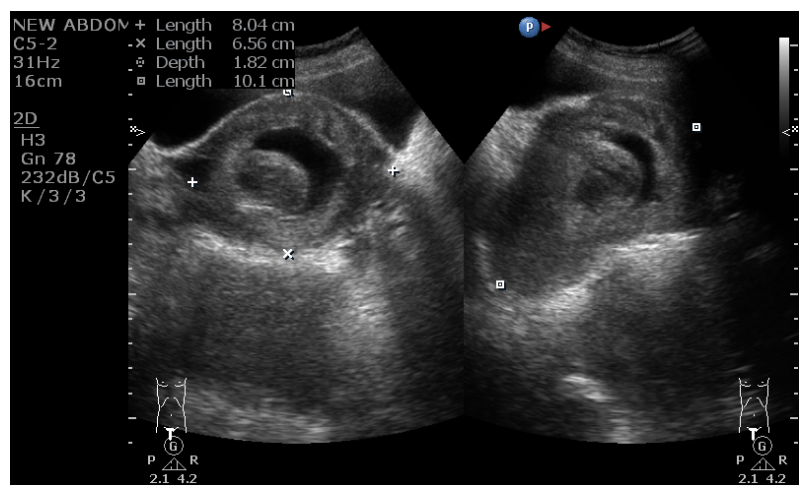

Figure 1. Ultrasound image showing the bulky uterus with mass lesion inside the endometrial cavity (arrow).

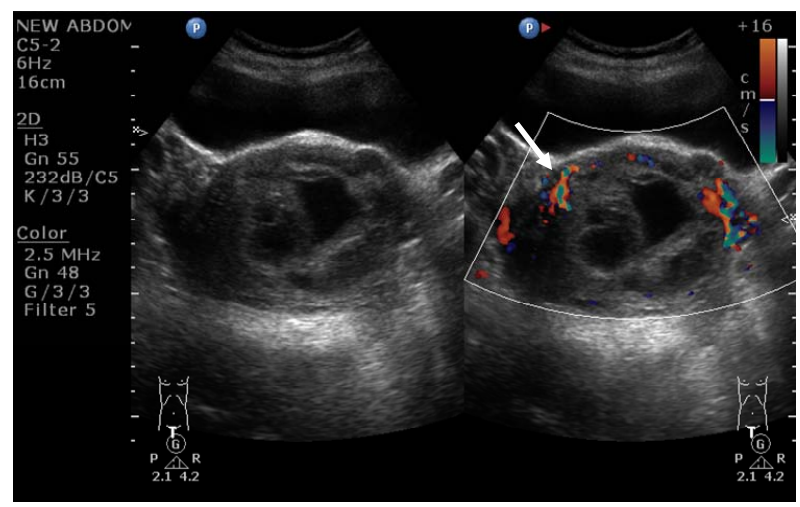

Figure 2. Doppler image showing the vascularity of the lesion (arrow).

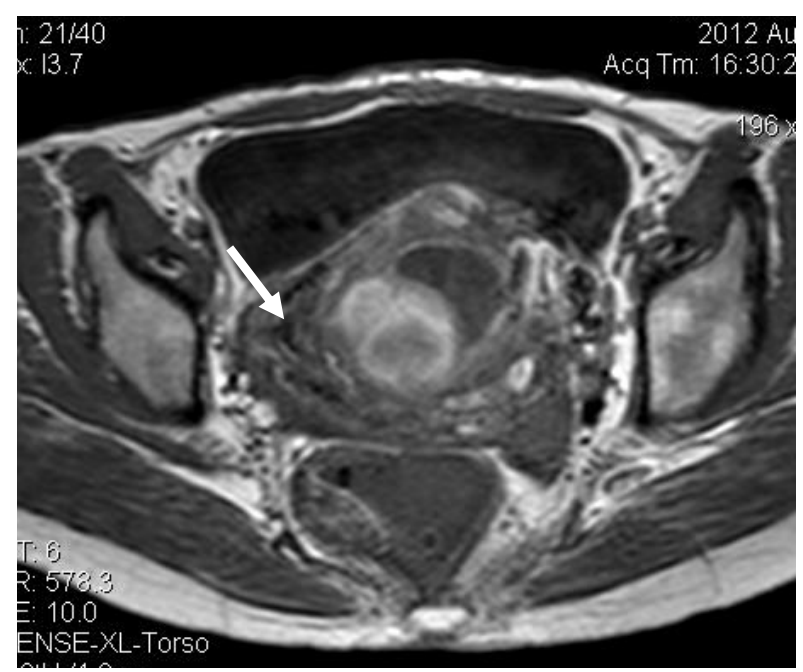

Figure 3. T1W axial image showing the lesion within the endometrial cavity with multiple flow voids (arrow).

MRI, the diagnosis of GTN i.e. Invasive mole was considered.

The patient was started on cycles of Inj. Methotrexate with Folinic acid, till near normalisation of the B hCG levels. There was persistence of the per vaginal bleeding and patient needed repeated blood transfusion for treat

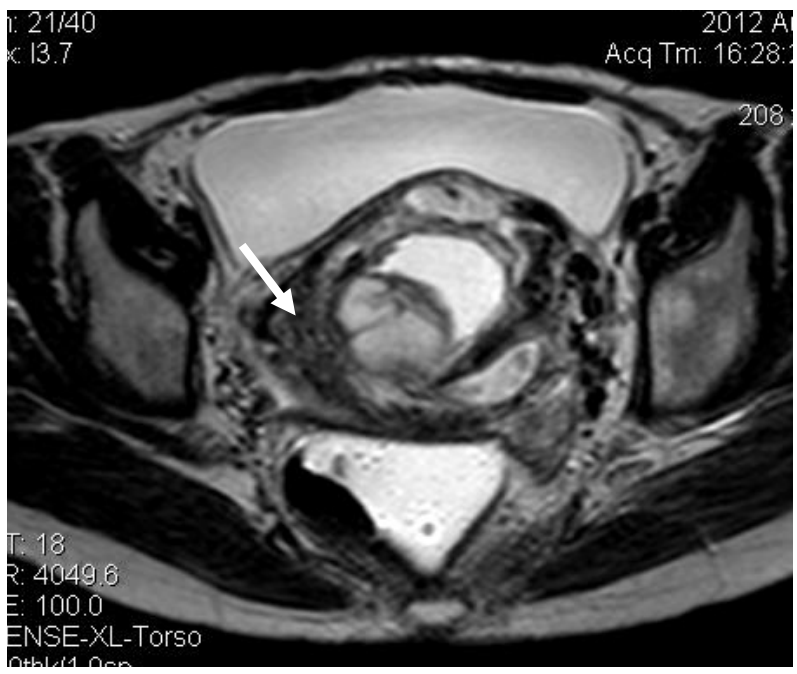

Figure 4. $\mathrm{T} 2 \mathrm{~W}$ axial image showing the solid cystic lesion within the endometrial cavity along with multiple flow voids (arrow).

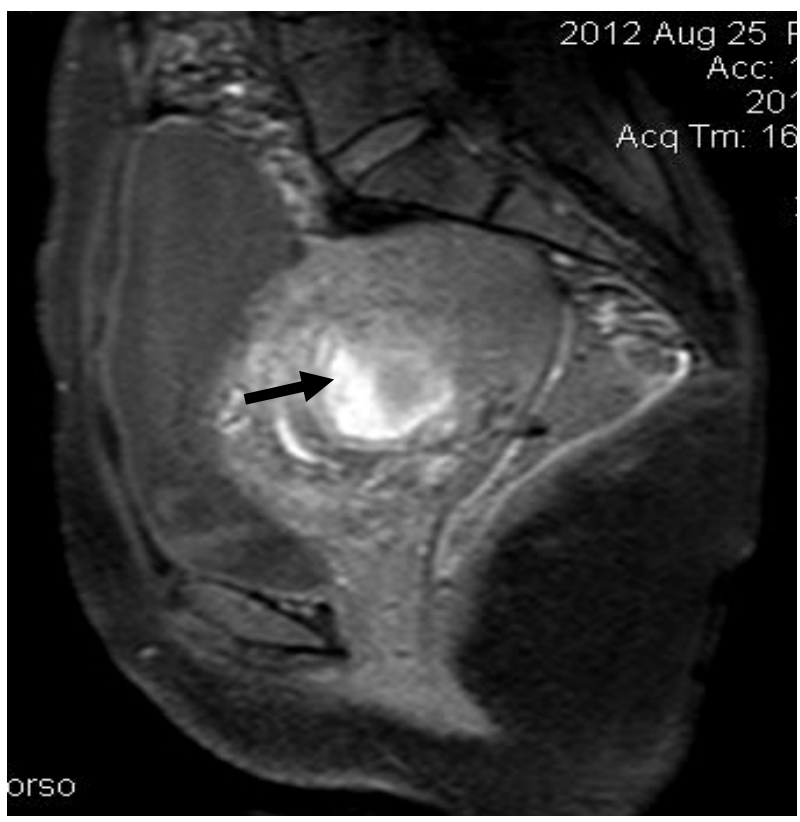

Figure 5. Pre contrast (gadolinium) sagittal T1W SPIR image showing the heterogeneous appearance of the lesion (arrow) distending the endometrial cavity.

ment of her anemia. Repeat pelvic US examination showed residual lesion with nearly unchanged vascularity (Figures 8 and 9). The patient was then referred to Interventional Radiology department for bilateral selective uterine artery embolization. After stabilisation of the vital parameters, patient was taken for uterine artery angiography. Using Seldinger's method, via right transfemoral approach, selective angiography of left uterine artery was performed using of $5 \mathrm{~F}$ Robert's uterine catheter (cordis), it showed high flow arterio-venous fistula with multiple tortuous feeding arteries and resultant early 


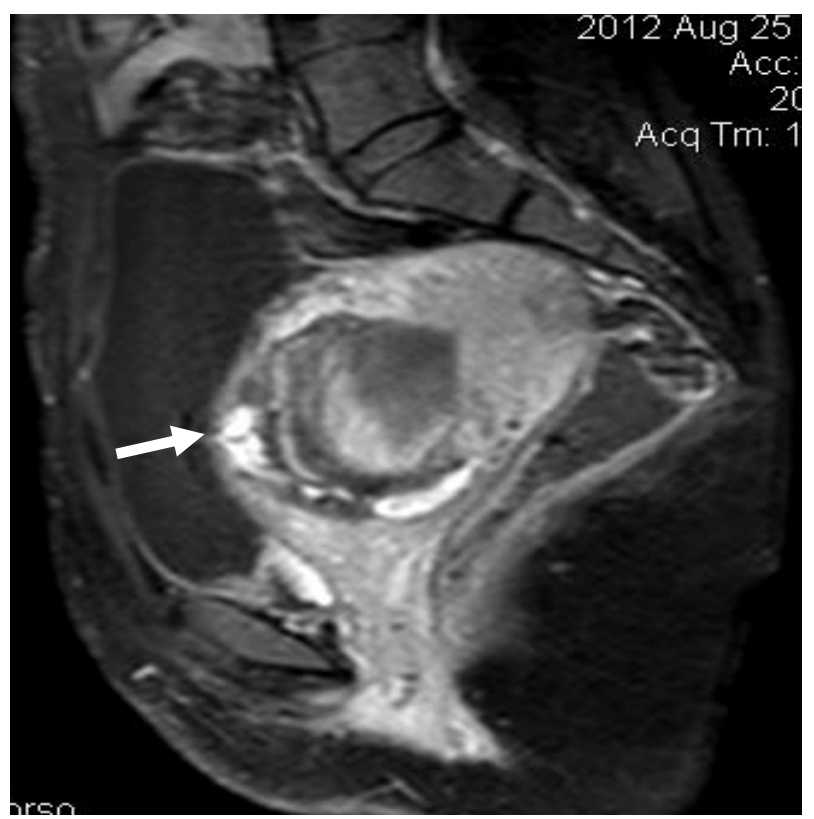

Figure 6. Post contrast (gadolinium) sagittal T1W SPIR image showing the heterogeneous enhancement of the lesion (arrow).

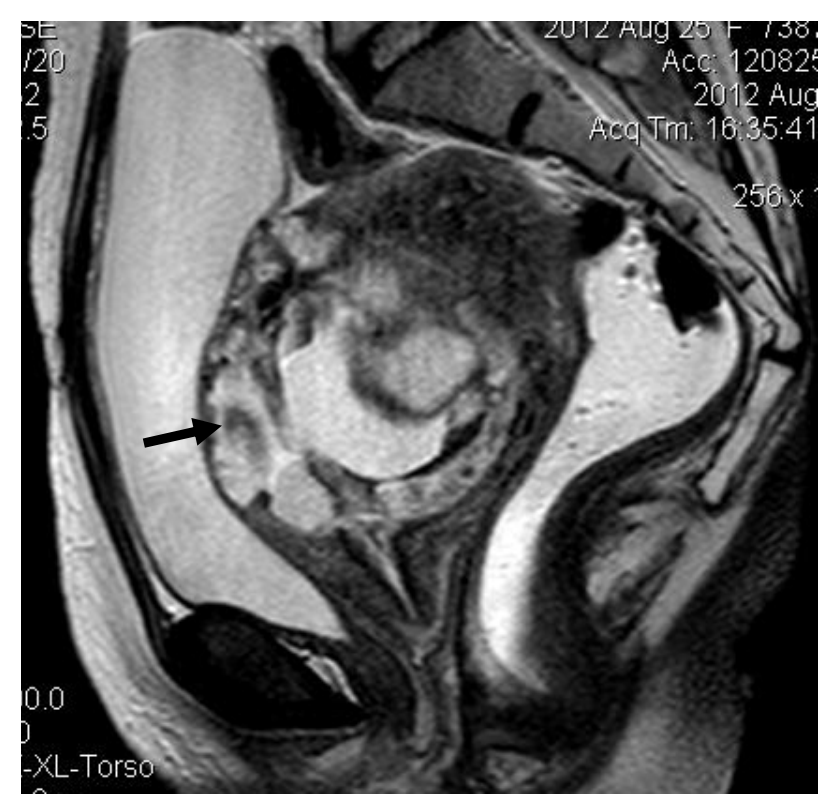

Figure 7. T2W sagittal image showing the bulky uterus with the lesion inside with invasion of uterine wall by the lesion at multiple places (arrow).

filling of the veins (Figures $\mathbf{1 0}$ and 11). In this ituation, glue embolization was considered to be ideal to close the high flow fistula. Through Robert's uterine catheter, microcathetor (Miraflex, 2.5 F) introduced into the left

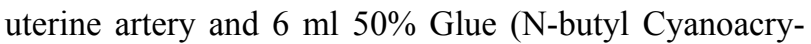
late) mixed with lipoidol in equal proportion injected under DSA guidance with catheter tip in the feeder arteries. The adequacy of the procedure was checked by post embolization check angiogram which revealed no

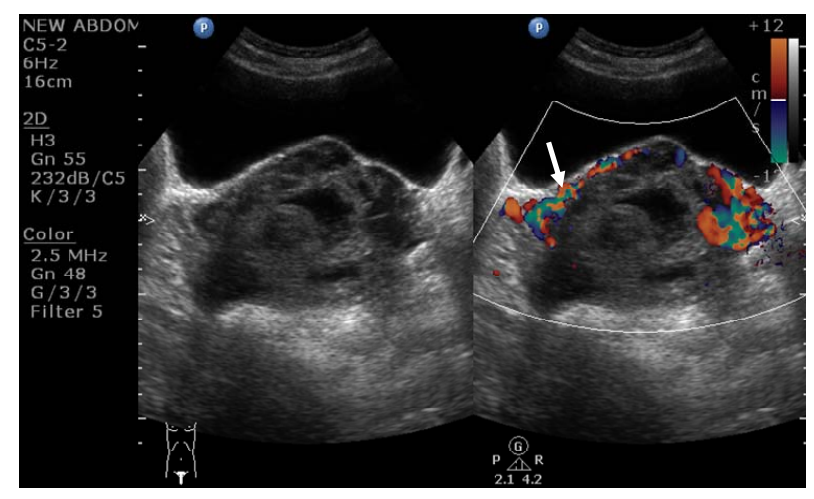

Figure 8. Post methotrexate ultrasound image showing the residual lesion and the unchanged vascularity (arrow).

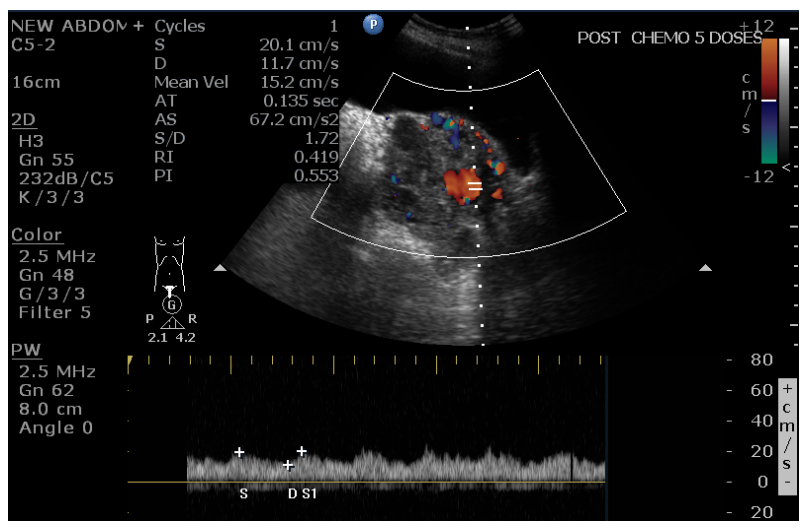

Figure 9. Post methotrexate ultrasound image showing unchanged vascularity with low resistivity index inside the lesion.

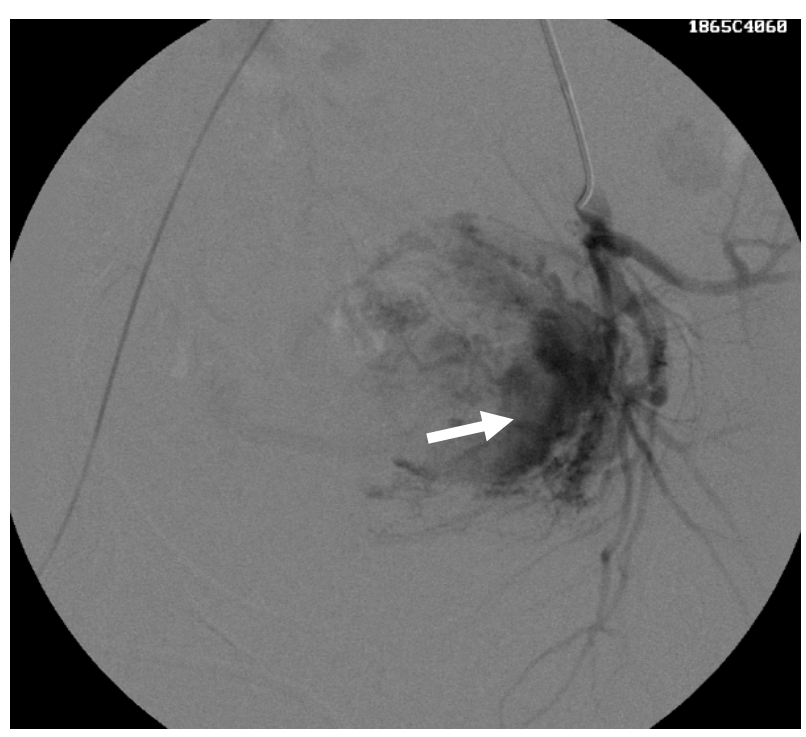

Figure 10. Left uterine angiogram showing the abnormal vascular blush (white arrow) with tortuous feeding arterial supply.

residual abnormal vascular blush (Figure 12). Then, the right side uterine artery was cannulated with the same Robert's uterine catheter and angiogram performed. Here too, there was significant abnormal vascular blush, 


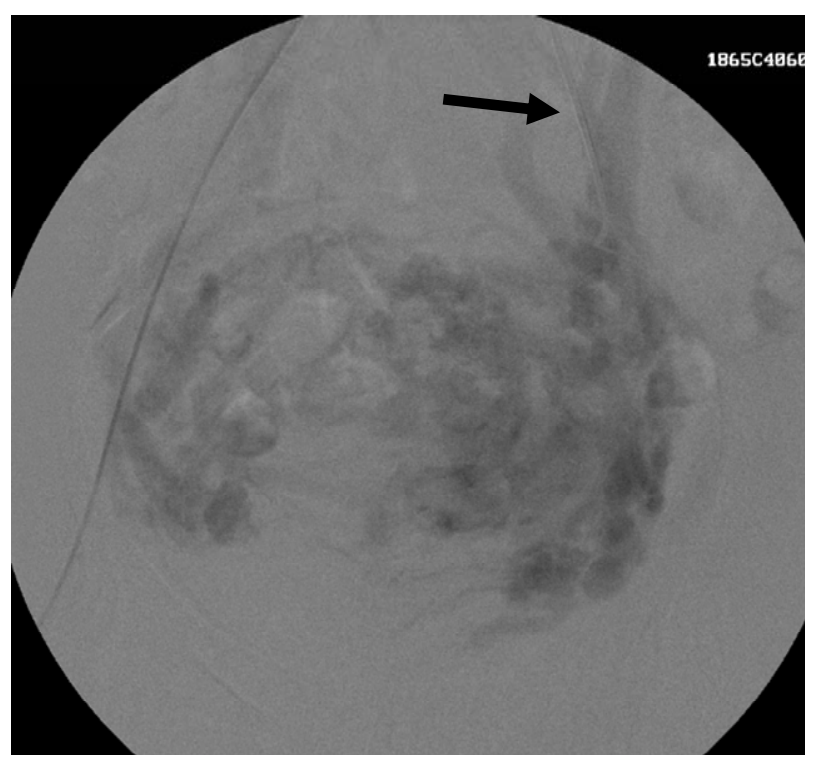

Figure 11. Left uterine angiogram showing the abnormal vascular blush and early filling of the veins (black arrow).

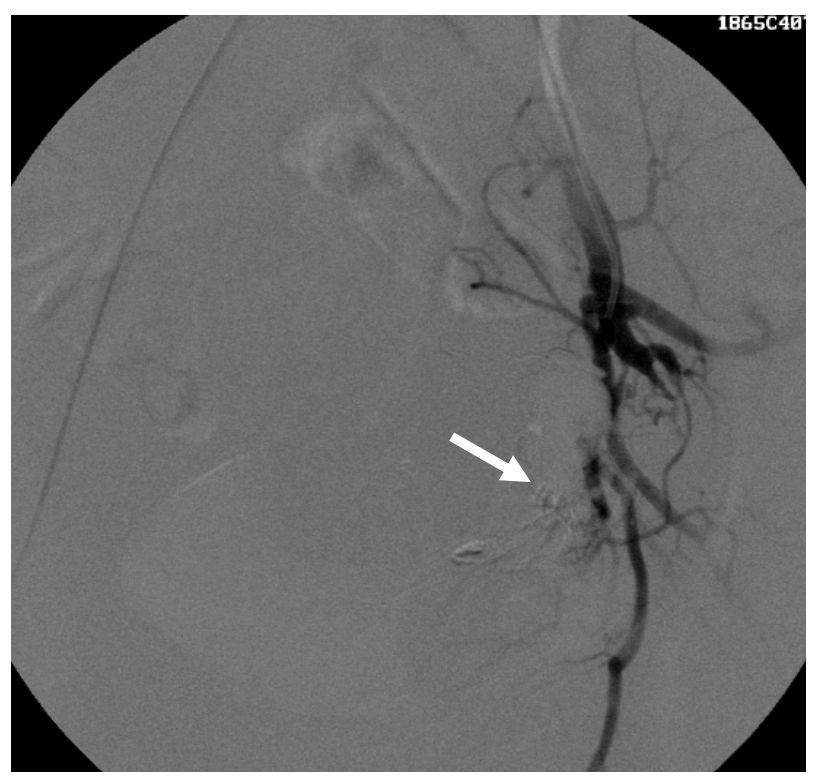

Figure 12. Post embolization check angiogram showing the glue cast (arrow) in situ with absence of the abnormal vascular blush.

but comparatively lesser than other side (Figure 13). Hence, here embolization with Polyvinyl Alcohol Particles was chosen as the correct option. Under DSA guidance, PVA particles of size 300 - 500 um mixed with iodinated contrast was injected. Post embolization check angiogram was performed to see for adequacy of the procedure (Figure 14). The patient was kept indoor for 5 days after the embolization procedure to see for any complications. Except for abdominal pain, nausea and vomiting, the patient did not have any other major complaints in the recovery period. Immediate post emboliza-

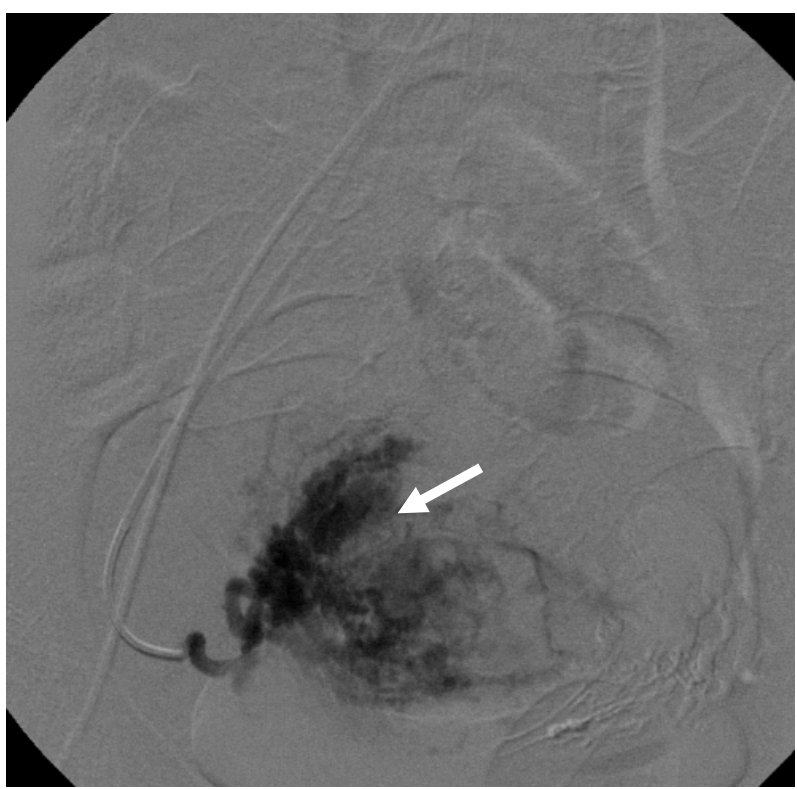

Figure 13. Right uterine artery angiogram showing the abnormal vascular blush (arrow).

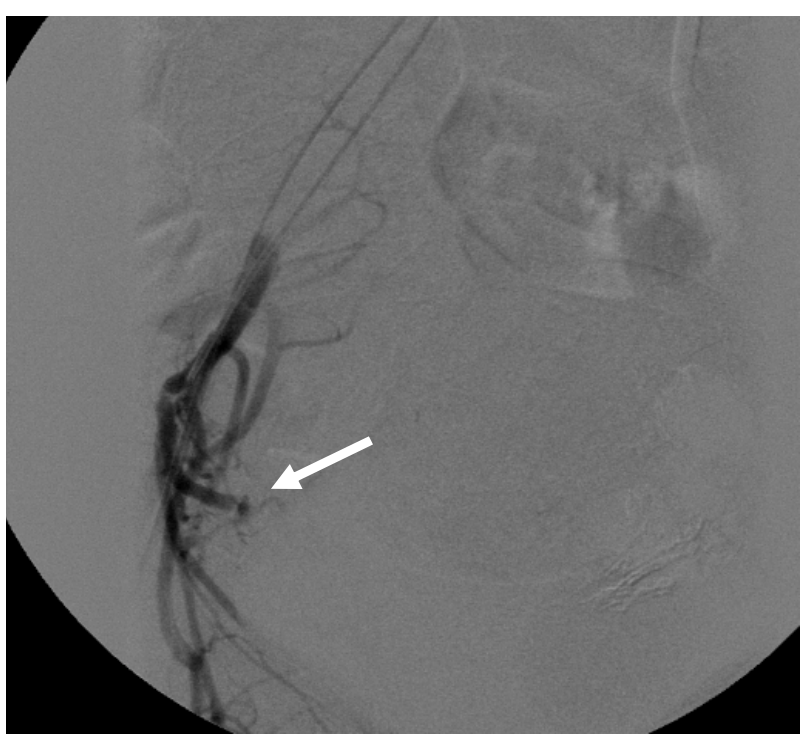

Figure 14. Post embolization check angiogram showing the absence of the abnormal blush (arrow).

tion ultrasound study revealed absence of the abnormalvascularity (Figure 15). There was improvement in the patient's clinical status with immediate stoppage of the per vaginal bleeding and rapid improvement in the patient's laboratory parameters, she was then discharged. The patient is kept on regular follow up and her B hCG levels being monitored which were within normal limits till now. She is now advised contraception up to the completion of follow up period.

\section{DISCUSSION}

Gestational trophoblastic diseases (GTD) and neoplasias 


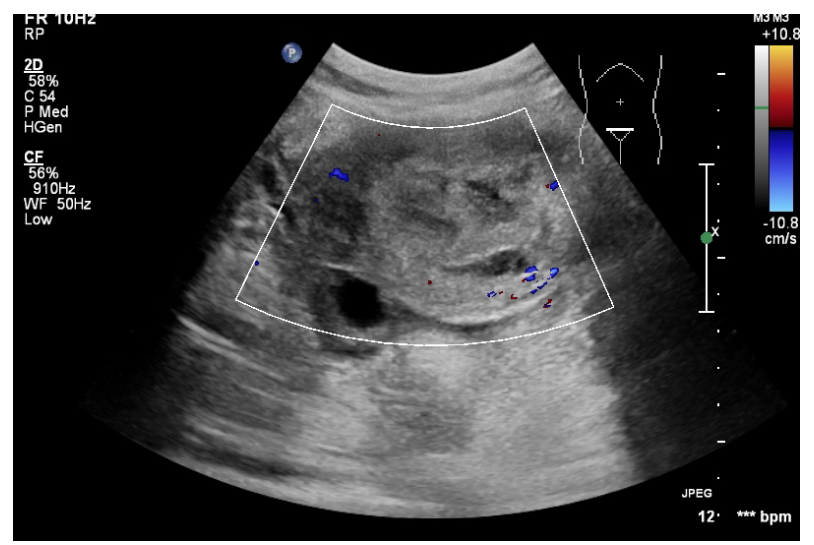

Figure 15. Immediate post embolisation ultrasound image showing the absence of the abnormal vascularity.

(GTN) are classified as follows [5]:

- Hydatidiform mole (HM).

o Complete HM.

o Partial HM.

- Invasive mole (chorioadenoma destruens).

- GTN.

o Choriocarcinoma.

o Placental-site Trophoblastic tumor (PSTT; very rare).

o Epitheliod Trophoblastic Tumor (ETT; even more rare).

Hydatidiform mole shows gross cyst-like swellings of the chorionic villi that are caused by an accumulation of fluid. The villous core shows disintegration and loss of blood vessels.

When an ovum that has extruded its maternal nucleus is fertilized by either a single sperm, with subsequent chromosome duplication, or two sperm, resulting in either case in a diploid karyotype, which is mostly $46 \mathrm{XX}$ or rarely $46 \mathrm{XY}$, a complete mole is formed. Complete mole does not show fetal parts or amniotic fluid. The risk of developing GTN in such complete moles is about 15 to $25 \%$.

A partial mole is formed when the ovum retains its nucleus but is fertilized by a single sperm, with subsequent chromosome duplication, or is fertilized by two sperm; with the possible resulting triploid karyotypes being $69 \mathrm{XXY}, 69 \mathrm{XXX}$, or $69 \mathrm{XYY}$. It may show fetal parts or amiotic fluid. The ultrasound diagnosis of a partial molar pregnancy is more complex; the finding of multiple soft markers, including both cystic spaces in the placenta and a ratio of transverse to antero-posterior dimension of the gestation sac of greater than 1.5, is required for the reliable diagnosis of a partial molar pregnancy. Transformation into GTN is much more rare $(<5 \%)$ in cases of partial moles.

Invasive moles are locally invasive, more aggressive than the hydatidiform moles but less than choriocarci- nomas. Microscopically, they show trophoblastic invasion of the myometrium, and even the parametrium, blood vessels. The initial radiological investigation of choice is the pelvic ultrasound, which shows bulky uterus with an echogenic vascular mass invading the myometrium. Colour Doppler study shows high velocity, low impedence flow. Soto-Wright et al. demonstrated a reduction in the mean gestation at presentation from 16 weeks, during the time period $1965-1975$, to 12 weeks between 1988-1993 [6]. The majority of histologically proven complete moles are associated with an ultrasound diagnosis of delayed miscarriage or anembryonic pregnancy $[7,8]$. In one study, the accuracy of pre-evacuation diagnosis of molar pregnancy increased with increasing gestational age, $35 \%-40 \%$ before 14 weeks increasing to $60 \%$ after 14 weeks [8]. A further study suggested a $56 \%$ detection rate for ultrasound examination. Estimation of B hCG levels may be of value in diagnosing molar pregnancies: B hCG levels greater than two multiples of the median may help [9]. MRI pelvis is done to see the extent of invasion and local spread of the lesion. It shows a poorly defined mass that invades the myometrium at places to a variable extent. Complete or partial disruption of the junctional zone may also be seen.

Typical signal characteristics include:

- T1 W sequences: usually iso-intense to the myometrium with scattered foci of high signal intensity (from the presence of haemorrhage).

- $\mathrm{T} 2 \mathrm{~W}$ sequences: mixed signal intensity due to areas of haemorrhages, cystic and necrotic changes.

The post-contrast enhancement is variable.

Choriocarcinoma is a malignant tumour of the trophoblastic epithelium. The tumor invades the uterine muscles and blood vessels with areas of hemorrhages and necrosis within. The tumour usually shows distant metastatic lesions which are hyper vascular, the most common sites of which are lungs, brain, liver, pelvis, vagina, spleen, intestines, and kidney. Mostly they follow an HM pregnancy, spontaneous abortion, or ectopic pregnancy; but about one-quarter are preceded by a fullterm pregnancy.

Placental-site trophoblastic tumor is a very rare tumor arising from the placental implantation site. The malignant cells infiltrate the myometrium, and there is vascular invasion. Elevations in serum B hCG levels are relatively low and unreliable $[5,10]$ compared with the marked elevations seen in choriocarcinoma. In contrast to choriocarcinoma, PSTT have much lower growth rates, and presentation after a full-term pregnancy is often delayed by months or years. They are generally resistant to chemotherapy [11]. Therefore, hysterectomy is the standard primary treatment if the tumour is confined to the uterus. However, about $35 \%$ of PSTTs have distant metastases at diagnosis $[5,10]$. 
Epitheliod Trophoblastic Tumor is an extremely rare trophoblastic tumour [12,13]. It appears to be less aggressive than choriocarcinoma. It may show varying clinical behaviour ranging from benign to malignant. However, about one-third of patients present with metastases, usually in the lungs.

\subsection{Treatment of Gestational Trophoblastic Neoplasia}

\subsubsection{Suction Curettage}

Suction curettage is the method of choice of evacuation for complete molar pregnancies.

Suction curettage is also the method of choice of evacuation for partial molar pregnancies except when the size of the fetal parts deters the use of suction curettage where medical evacuation can be used.

\subsubsection{Chemotherapy}

Treatment used is based on the FIGO 2000 scoring system for GTN [14], which is described in Table 1. Women with scores $\leq 6$ are at low risk and treated with single-agent intramuscular methotrexate alternating daily with folinic acid for 1 week followed by 6 rest days. Women with scores $\geq 7$ are at high risk and are treated with intravenous multi-agent chemotherapy, which includes combinations of methotrexate, dactinomycin, etoposide, cyclophosphamide and vincristine. Treatment is continued, in all cases, until the B hCG level has returned to normal and then for a further 6 consecutive weeks.

The cure rate for women with a score $\leq 6$ is almost $100 \%$; the rate for women with a score $\geq 7$ is $95 \%$.

\subsubsection{Uterine Artery Embolization}

Uterine vascular malformations persist in $10 \%-15 \%$ of patients with gestational trophoblastic neoplasias, even after complete remission of the tumour following chemotherapy, and the associated symptom being per vaginal bleeding. Since the majority of such vascular mal- formations are supplied predominantly by the uterine arteries, bilateral selective uterine artery angiography and embolization is the minimally invasive and more effective treatment modality of choice as an alternative to surgical treatment $[15,16]$. In case of recurrence due to collateral formation, re-embolization can be done.

The main side effect of embolization is pelvic pain, and is easily controlled by analgesics, which is often seen in cases of uterine artery embolization for other causes [17]. The other potential side effects include buttock and lower limb claudication due to extensive and multiple embolization in the pelvic vessels. Rare complications such as perineal skin sloughing, utero-vaginal and recto-vesico-vaginal fistulae, and bladder necrosis, have also been reported in past where the internal iliac arteries have been embolized with cryano-acrylate as the embolizing agent [18-20].

Embolization of the uterine arteries has not been associated with uterine infarction because of the presence of a rich collateral vascular network within the pelvis [21]. There is further evidence that arterial recanalization sufficient to sustain the uterus through a full-term gestation can occur after uterine artery embolization for vascular malformations associated with gestational trophoblastic neoplasias [22,23].

\subsubsection{Surgical Hysterectomy}

Hysterectomy is done in cases of uncontrolled uterine bleeding, placental site trophoblastic tumors as they are not responsive to chemotherapy and when uterine artery embolization is not possible.

\subsection{Follow up}

Follow up of the patient is usually required for a period of 6 months from normalisation of the B hCG levels.

All women should notify the screening centre at the end of any future pregnancy, whatever the outcome of the pregnancy. B hCG levels are measured 6 - 8 weeks after the end of the pregnancy to exclude disease recurrenc.

Table 1. FIGO 2000 scoring system for GTN.

\begin{tabular}{|c|c|c|c|c|}
\hline FIGO SCORING SYSTEM & $\mathbf{0}$ & 1 & 2 & 4 \\
\hline Age (years) & $<40$ & $\geq 40$ & - & - \\
\hline Antecedent pregnancy & Mole & Abortion & Term & - \\
\hline Interval months from end of index pregnancy to treatment & $<4$ & $4-7$ & $7-13$ & $\geq 13$ \\
\hline Pretreatment serum B hCG (IU/L) & $<10^{3}$ & $10^{3}-10^{4}$ & $10^{4}-10^{5}$ & $\geq 10^{5}$ \\
\hline Largest tumor size, including uterus $(\mathrm{cm})$ & $<3$ & $3-5$ & $\geq 5$ & - \\
\hline Site of metastases & Lung & Spleen, kidney & Gastro-intestinal & Liver, brain \\
\hline Number of metastases & - & $1-4$ & $5-8$ & $>8$ \\
\hline Previous failed chemotherapy & - & - & Single drug & 2 or more drugs \\
\hline
\end{tabular}


Two large case series of nearly 9000 cases have shown that, once B hCG has normalised, the possibility of GTN developing is very low $[24,25]$.

Women should be advised not to conceive until their follow-up is complete. Women who undergo chemo-therapy are advised not to conceive for at least 1 year after completion of treatment.

\section{CONCLUSION}

In conclusion, selective uterine artery embolization is a safe, minimally invasive and effective treatment with lesser morbidity for uncontrolled haemorrhage from residual uterine vascular malformations even after chemotherapy completion in patients with gestational trophoblastic neoplasias. Although repeat embolotherapy for recurrent bleeding may be required, hysterectomy can be avoided and fertility preserved, which is extremely important for this group of patients.

\section{REFERENCES}

[1] Lim, A.K.P., Agarwal, R., Seckl, M.J., Newlands, E.S., Barrett, N.K. and Mitchell, A.W.M. (2000) Embolization of bleeding residual uterine vascular malformations in patients with treated gestational trophoblastic tumors. RSNA Scientific Assembly.

[2] Bagshawe, K.D., Dent, J. and Webb, J. (1986) Hydatidiform mole in England and Wales 1973-1983. Lancet, 2, 673-677. doi:10.1016/S0140-6736(86)90179-0

[3] Bagshawe, K.D., Dent, J., Newlands, E.S., Begent, R.H. and Rustin, G.J. (1989) The role of low-dose methotrexate and folinic acid in gestational trophoblastic tumours (GTT). British Journal of Obstetrics and Gynaecology, 96, 795-802. doi:10.1111/j.1471-0528.1989.tb03318.x

[4] Newlands, E.S., Bagshawe, K.D., Begent, R.H.J., Rustin, G.J.S., Holden, L. and Dent, J. (1986) Developments in chemotherapy for medium and high risk patients with gestational trophoblastic tumours. British Journal of $\mathrm{Ob}$ stetrics and Gynaecology, 93, 63-69.

[5] Lurain, J.R. (1990) Gestational trophoblastic tumors. Seminars in Surgical Oncology, 6, 347-353. doi:10.1002/ssu.2980060610

[6] Soto-Wright, V., Berstein, M., Goldstein, D.P. and Berkowitz, R.S. (1995) The changing clinical presentation of complete molar pregnancy. Obstetrics and Gynaecology, 86, 775-779. doi:10.1016/0029-7844(95)00268-V

[7] Sebire, N.J., Rees, H., Paradinas, F., Seck, M. and Newlands, E.S. (2001) The diagnostic implications of routine ultrasound examination in histologically confirmed early molar pregnancies. Ultrasound in Obstetrics \& Gynecology, 18, 662-665.

[8] Fowler, D.J., Lindsay, I., Seck, M.J. and Sebire, N.J. (2006) Routine pre-evacuation ultrasound diagnosis of hydatidiform mole: Experience of more than 1000 cases from a regional referral center. Ultrasound in Obstetrics \& Gynecology, 27, 56-60. doi:10.1002/uog.2592
[9] Johns, J., Greenwold, N., Buckley, S. and Jauniaux, E. (2005) A prospective study of ultrasound screening for molar pregnancies in missed miscarriages. Ultrasound in Obstetrics \& Gynecology, 25, 493-497. doi:10.1002/uog. 1888

[10] Feltmate, C.M., Genest, D.R., Goldstein, D.P., et al. (2002) Advances in the understanding of placental site trophoblastic tumor. Journal of Reproductive Medicine, 47, 337 341.

[11] Schmid, P., Nagai, Y., Agarwa, R., et al. (2009) Prognostic markers and long-term outcome of placental-site trophoblastic tumours: A retrospective observational study. Lancet, 374, 48-55. doi:10.1016/S0140-6736(09)60618-8

[12] Shih, I.M. and Kurman, R.J. (1998) Epithelioid trophoblastic tumor: A neoplasm distinct from choriocarcinoma and placental site trophoblastic tumor simulating carcinoma. The American Journal of Surgical Pathology, 22, 1393-1403. doi:10.1097/00000478-199811000-00010

[13] Palmer, J.E., Macdonald, M., Wells, M., et al. (2008) Epithelioid trophoblastic tumor: A review of the literature. Journal of Reproductive Medicine, 53, 465-475.

[14] International Federation of Obstetrics and Gynecology Oncology Committee (2002) FIGO staging for gestational trophoblastic neoplasia 2000. International Journal of Gynecology \& Obstetrics, 77, 285-287. doi:10.1016/S0020-7292(02)00063-2

[15] Antebi, E., Adar, R., Deutsch, V. and Mozes, M. (1974) Arteriovenous fistula complicating gynecological operations. Obstetrics and Gynaecology, 44, 858-861.

[16] De Cockshott, P.V. and Hendrickse, J.P. (1967) Persistent arteriovenous fistulae following chemotherapy of malignnant trophoblastic disease. Radiology, 88, 329-333.

[17] Vashisht, A., Studd, J.W., Carey, A.H., et al. (2000) Fibroid embolization: A technique not without significant complications. British Journal of Obstetrics and Gynaecology, 107, 1166-1170. doi:10.1111/j.1471-0528.2000.tb11119.x

[18] Hare, W.S.C. and Holland, C.J. (1983) Paresis following internal iliac artery embolization. Radiology, 46, 47-51.

[19] Lang, E.K. (1989) Management of haemorrhaging pelvic neoplasms by transcatheter embolization. Journal of Vascular and Interventional Radiology, 4, 113-117.

[20] Lang, E.K. (1981) Transcatheter embolization of pelvic vessels for control of hemorrhage. Radiology, 140, 331339.

[21] Belli, A.M., Hemingway, A.P., Neal, F.E. and Millar, D.R. (1989) Arteriovenous malformation of the uterus related to trophoblastic disease: A case for surgery or embolization? Journal of Vascular and Interventional Radiology, 4, 112-118.

[22] Chapman, D.R. and Lutz, M.H. (1985) Report of a successful delivery after nonsurgical management of a choriocarcinoma-related pelvic arteriovenous fistula. American Journal of Obstetrics \& Gynecology, 153, 155-157.

[23] McIvor, J. and Cameron, E.W. (1996) Pregnancy after uterine embolization to control haemorrhage from gestational trophoblastic tumours. British Journal of Radiology, 69, 624-629. doi:10.1259/0007-1285-69-823-624 
[24] Tham, B.W.L., Everard, J.E., Tidy, J.A., Drew, D. and Hancock, B.W. (2003) Gestational trophoblastic disease in the Asian population of Northern England and North Wales. British Journal of Obstetrics and Gynaecology, 110, 555-559. doi:10.1046/j.1471-0528.2003.01413.x

[25] Pisal, N., Tidy, J. and Hancock, B. (2004) Gestational trophoblastic disease: Is intensive follow up essential in all women? British Journal of Obstetrics and Gynaecology, 111, 1449-1451.

doi:10.1111/j.1471-0528.2004.00339.x

[26] Sebire, N.J., Foskett, M., Short, D., Savage, P., Stewart, W., Thomson, M., et al. (2007) Shortened duration of human chorionic gonadotrophin surveillance following complete or partial hydatidiform mole: Evidence for revised protocol of a UK regional trophoblastic disease unit. British Journal of Obstetrics and Gynaecology, 114, 760762. doi:10.1111/j.1471-0528.2007.01320.x

[27] Fine, C., Bundy, A.L., Berkowitz, R., Boswell, S.B., Berezin, A.F. and Doubilet, P.M. (1989) Sonographic diagnosis of partial hydatidiform mole. Obstetrics and Gynae- cology, 73, 414-418.

[28] Benson, C.B., Genest, D.R., Bernstein, M.R., SotoWright, V., Goldstein, D.P. and Berkowitz, R.S. (2000) Sonographic appearance of first trimester complete hydatidiform moles. Journal of Ultrasound in Obstetrics \& Gynecology, 16, 188-191. doi:10.1046/j.1469-0705.2000.00201.x

[29] Newlands, E.S. (2003) Presentation and management of persistent gestational trophoblastic disease and gestational trophoblastic tumours in the UK. In: Hancock, B.W., Newlands, E.S., Berkowitz, R.S. and Cole, L.A., Eds., Gestational Trophoblastic Disease. 3rd Edition, International Society for the Study of Trophoblastic Disease, London, 277-298. www.isstd.org/isstd/book.html

[30] Woolas, R.P., Bower, M., Newlands, E.S., Seck, M.J., Short, D. and Holden, L. (1998) Influence of chemotherapy for gestational trophoblastic disease on subsequent pregnancy outcome. British Journal of Obstetrics and Gynaecology, 105, 1032-1035. doi:10.1111/j.1471-0528.1998.tb10271.x 